\title{
Evaluation of the Combined Effect of Mobility and Seasonality on the COVID-19 Pandemic: A Lombardy-Based Study
}

\section{Yuri Falzone}

Neurology Unit, IRCCS San Raffaele Scientific Institute, Milan, Italy

\section{Luca Bosco}

Neurology Unit, IRCCS San Raffaele Scientific Institute, Milan, Italy

\section{Giacomo Sferruzza}

Neurology Unit, IRCCS San Raffaele Scientific Institute, Milan, Italy

\section{Tommaso Russo}

Neurology Unit, IRCCS San Raffaele Scientific Institute, Milan, Italy

\section{Marco Vabanesi}

Neurology Unit, IRCCS San Raffaele Scientific Institute, Milan, Italy

Massimo Filippi ( $\nabla$ filippi.massimo@hsr.it)

Neurology Unit, IRCCS San Raffaele Scientific Institute, Milan, Italy

\section{Research Article}

Keywords: SARS-CoV-2, UV, environmental factors, transmissibility, GAM analysis

Posted Date: March 23rd, 2021

DOI: https://doi.org/10.21203/rs.3.rs-315449/v1

License: (1) This work is licensed under a Creative Commons Attribution 4.0 International License. Read Full License 


\section{Abstract}

Restrictions to human mobility had a significant role in limiting SARS-CoV-2 spread. It has been suggested that seasonality might affect viral transmissibility. Our study retrospectively investigates the combined effect that seasonal environmental factors and human mobility played on transmissibility of SARS-CoV-2 in Lombardy, Italy, in 2020.

Environmental data were collected from accredited open-source web services. Aggregated mobility data for different points of interests were collected from Google Community Reports. The Reproduction number $\left(R_{t}\right)$, based on the weekly counts of confirmed symptomatic COVID-19, non-imported cases, was used as a proxy for SARS-CoV-2 transmissibility. Assuming a non-linear correlation between selected variables, we used a Generalized Additive Model (GAM) to investigate with univariate and multivariate analyses the association between seasonal environmental factors (UV-index, temperature, humidity, and atmospheric pressure), location-specific mobility indices, and $\mathrm{R}_{\mathrm{t}}$.

UV-index was the most effective environmental variable in predicting $\mathrm{R}_{\mathrm{t}}$. An optimal two-week lag-effect between changes in explanatory variables and $R_{t}$ was selected. The association between $R_{t}$ variations and individually taken mobility indices differed: Grocery \& Pharmacy, Transit Station and Workplaces displayed the best performances in predicting $\mathrm{R}_{\mathrm{t}}$ when individually added to the multivariate model together with UV-index, accounting for $85.0 \%, 85.5 \%$ and $82.6 \%$ of $\mathrm{R}_{t}$ variance, respectively. According to our results, both seasonality and social interaction policies played a significant role in curbing the pandemic. Non-linear models including UV-index and location-specific mobility indices can predict a considerable amount of SARS-CoV-2 transmissibility in Lombardy during 2020, emphasizing the importance of social distancing policies to keep viral transmissibility under control, especially during colder months.

\section{Introduction}

The novel severe acute respiratory syndrome coronavirus 2 (SARS-CoV-2), causative agent of coronavirus disease 2019 (COVID-19), emerged in Wuhan (China) in December 2019. COVID-19 rapidly spread worldwide, reaching the epidemiological criteria to be declared a pandemic by the World Health Organization (WHO) in March 2020.[1]

During 2020, the COVID-19 pandemic had a striking impact not only on healthcare systems, but also on social politics and welfares. Lombardy (Italy), one of the most populated regions in Europe, was among the first ones suffering the whirlwind effects of the pandemic in the Western world, with a burden of infections that overwhelmed the healthcare systems capacities.[2] To contain the pandemic, governments worldwide applied different non-pharmaceutical interventions in order to reduce SARS-CoV-2 transmissibility. In Lombardy, strict restrictions to mobility and social interaction were imposed, with aggregate mobility data displaying a relevant drop during spring.[3] Several studies have already 
established the crucial role that social distancing policies, restrictions to individuals mobility patterns, and non-pharmaceutical interventions as a whole have played in slowing the spreading of the virus.[4-6]

During late spring 2020, many countries in the Northern hemisphere loosened these restrictions, due to a sustained reduction in viral transmissibility and a lower pressure on healthcare systems. This led some authors to question the eventuality of a "second wave" of the pandemic, during autumn.[7] Direct, dropletmediated, human-to-human transmission has been recognized as the main viral route of transmission. However, consistent evidence of susceptibility of SARS-CoV-2 to environmental factors, such as temperature, humidity, and simulated sunlight, has emerged from experimental data, proposing similarities with other viruses with a lipidic envelope.[8, 9] SARS-CoV-2 has proven itself highly resilient in saliva and other body fluids on surfaces outside the human body, making the putative role of environmental factors on its persistence potentially crucial in the transmission of the disease.[10]

Some studies have already investigated the role of environmental factors such as temperature, humidity, and UV-index on SARS-CoV-2 virulence at different latitudes, achieving mixed and nonconclusive results. [11-15] Most of these works have, however, some major limitations: 1) data analyzed were mostly derived from the first "pandemic wave", an early phase in which epidemic growth was unbalanced by low immunity, so that even strong environmental drivers were unlikely to affect transmissibility; 2) the reduction in social interactions determined by government-imposed non-pharmaceutical interventions worldwide has been rarely weighted alongside environmental drivers, therefore acting as a possible strong confounding factor; 3 ) testing and reporting of cases around the globe, and sometimes even inside the same country, has varied significantly, so that country-specific limitations of data quality may support inconsistent inferences on evidences about the role of climatic factors.[15] Now that the era of SARS-CoV-2 vaccines has begun, uncertainty about the duration of natural and vaccine-induced immunity prompts new efforts in clarifying viral transmission dynamics. Indeed, the aim of the current study is to retrospectively investigate the effects of seasonal environmental factors and social interaction data on transmissibility of SARS-CoV-2 in Lombardy, during 2020.

\section{Methods}

\section{DATA SOURCES}

In this retrospective study, we assessed data at regional level for Lombardy (Italy) during the period from May 18, 2020 to December 13, 2020. Epidemiological data, including number of new confirmed cases and estimates of the reproduction number $\left(\mathrm{R}_{\mathrm{t}}\right)$, were collected from the national deputed entity, Istituto Superiore di Sanità (ISS). $\mathrm{R}_{\mathrm{t}}$ estimate is based on the weekly counts of confirmed symptomatic COVID-19, non-imported cases, referring to when symptoms developed. $\mathrm{R}_{\mathrm{t}}$ represents the average number of secondary cases that would arise at a given time from a primary infected case if the conditions remained stable after that time.[16] Due to its calculation method, $R_{t}$ relates to a subgroup of cases and to a rolling time window of around one week, which accounts for the reporting delay between symptom onset and case notification.[17] Since daily transmissibility may be influenced by particular events or random daily 
variation, ISS reports the average $R_{t}$ value of the previous week (seven days, corresponding to one generation time).[17]

Mean daily values for UV-index, provided in a time series format, were collected from the Tropospheric Emission Monitoring Internet Service (TEMIS) archive, hosted by the Royal Netherlands Meteorological Institute (KNMI, http://www.temis.nl/uvradiation/UVarchive.html) as part of a project of the European Space Agency (ESA). TEMIS provides validated near-real time satellite-based UV-index and UV dose timeseries for different UV locations around the globe [18]. Ispra $\left(45.8^{\circ} \mathrm{N}, 8.6^{\circ} \mathrm{E}\right.$, Lombardy, Italy) cloudfree erythemal UV-index time series was adopted for this study.

Average temperature at 2 meters above ground surface, average atmospheric pressure at ground surface, and average of relative humidity at 2 meters height were collected from the NASA Prediction Of Worldwide Energy Resources (POWER) Data Access Viewer (DAV, http://power.larc.nasa.gov). The parameters in POWER Release-8 are provided on a global grid with a spatial resolution of $0.5^{\circ}$ latitude by $0.5^{\circ}$ longitude.

Aggregate and anonymized mobility data fluctuations were collected from Google Community Mobility Reports (https://www.google.com/covid19/mobility/). These datasets describe relative variation in individual mobility dynamics, affecting selected categories of Points Of Interest (POIs), when compared to "baseline-days", which represent normal values for a specific day of the week, averaged from the five-week period (Jan 3 - Feb 6, 2020). Changes are calculated using the same kind of aggregated and anonymized data used to show popular times for places in Google Maps. Google Community Mobility Reports locations are grouped within six categories: Residential, Retail \& Recreation, Grocery \& Pharmacy, Parks, Transit Stations, Workplaces, and Residential Areas. Specific POIs for each mobility index are listed in Supplementary Table 1. Country-and region-wise data for each parameter are available on a daily basis (since Feb 15, 2020).

\section{DATA SELECTION AND PROCESSING}

In accordance with $\mathrm{R}_{\mathrm{t}}$ calculation method, we aggregated daily environmental and mobility daily values on a weekly basis (Monday to Sunday) to account for the 30 weeks from May 18 to December 13, 2020.

Among mobility parameters provided by Google Community Mobility Reports, five were considered relevant to our analysis (Transit Stations, Retail \& Recreation, Workplaces, Grocery \& Pharmacies, Parks) as being specifically affected by the national government mobility restriction measures. Residential mobility was excluded, as positive variations in this index are actually indicative of decreased mobility, suggesting instead increased activities in locations around the home environment.

\section{DATA ANALYSIS}

Assuming a non-linear relation between the selected data, we used a Generalized Additive Model (GAM) to investigate with univariate and multivariate analyses the association between seasonality, mobility 
indices and $R_{t}$. Shapiro-Wilk test was performed in order to assess $R_{t}$ values deviation from normal distribution. $R_{t}$ frequency distribution is shown in Supplementary Figure 1. It has been demonstrated that $R_{t}$ follows a negative binomial probability distribution, converging to the Poisson distribution for large value of the parameter $k$ of the discrete probability distribution.[19] For this reason, logarithm link function was selected for predictive GAM. The model applied to investigate a univariate association between variables and the $R_{t}$ is described as follows:

$\log \left(R_{t i+l a g}\right)=a+f\left(x_{i}, k=8\right)+\delta \times \log \left(R_{t i-1}\right)$

where $a$ is the intercept, $f$ denotes the smoother function, based on the penalized smoothing splines, $x_{i}$ denotes the $\mathrm{i}^{\text {th }}$-week predictive variable, including environmental variables (temperature, UV-index, humidity, and atmospheric pressure) and mobility indexes. $K$ represents the number of delimiting knots of predictor variable in the GAM model. The term $\left(\delta \times \log \left(\mathrm{Rt}_{\mathrm{i}-1}\right)\right)$ was used to correct the autocorrelation of $\mathrm{R}_{\mathrm{t}}$ time series, as shown by the autocorrelation function (ACF) (Supplementary Figure 2). Different lageffects were evaluated for each variable to explore potential plausible biological association with $R_{t}$. Akaike's information criterion (AIC) was used to compare different models.

\subsubsection{Multivariate analysis of influences on $R_{t}$}

Due to the high collinearity among mobility indices (Supplementary Table 2), the impact of each selected index was evaluated individually to avoid the risk of type I error due to concurvity in GAM. Each mobility index was individually added to GAM together with UV-index, considering a preselected two-week lag, in order to explore the potential role of each mobility index in predicting $\mathrm{R}_{\mathrm{t}}$, adjusted for a seasonal variation. The model used to investigate the multivariate association between UV-index, mobility, and $R_{t}$ is described as follows:

$\log \left(R t_{i+2}\right)=a+f_{1}\left(M_{i}, k=8\right)+f_{2}\left(U V_{i}, k=8\right)+\delta \times \log \left(R t_{i+1}\right)$

where $\mathrm{M}$ represents the $\mathrm{i}^{\mathrm{th}}$-week mobility index and UV the $\mathrm{i}^{\text {th }}$-week average of UV-index. AIC score minimization was used to evaluate the impact of each mobility index in predicting $R_{t}$, keeping into account seasonality.

All statistical analyses in the study were performed with R statistical package (R Foundation for Statistical Computing, Vienna, Austria), version 4.0.3.

\section{Results}

A 30-week period was included in the analysis. A total of 358818 new confirmed cases were reported in the study period in Lombardy, accounting for the $3.56 \%$ of the total population and for the $80.87 \%$ of all confirmed cases since the first documented case in Lombardy, in February 20, 2020. $R_{t}$ ranged from 0.52 [95\% C.I.: $0.22-1.21]$ to 1.17 [0.86-1.48] for the entirety of summer; it steeply increased during the 
transition to autumn and reached its peak at 2.09 [1.77-2.30] in the third week of October 2020 (Fig. 1a). Mean weekly UV-index and temperatures ranged from 0.87 to 9.02 and from $0.70^{\circ} \mathrm{C}$ to $25.04^{\circ} \mathrm{C}$, respectively. UV-index reached its peak in the last week of June, four weeks before the highest weekly temperature recorded value (Fig. 1b). Humidity and atmospheric pressure trends are displayed in Fig. 1c.

All mobility indices included in the analysis show a reduction when compared to baseline, except for Parks. In particular, Retail \& Recreation and Transit Station ranged from $-11.86 \%$ to $-59.86 \%$ and from $21.57 \%$ to $-60.71 \%$ respectively, with the downward peak reached in the second week of November. Workplace and Grocery \& Pharmacy ranged from $-18.86 \%$ to $-51.14 \%$ and from $-5.86 \%$ to $-27.57 \%$ respectively, with the downward peak reached on the second week of August, concurrently with holiday season. Parks ranged from $+60.14 \%$ in the second week of September (the fact that baseline mobility was calculated in the month of January accounts for this result), to $-44.57 \%$ in the last week of October. After the loosening of mobility restrictions, following the end of the first pandemic wave, mobility curves kept a relatively stable profile during summer, except for a downward shift during holiday weeks in August. Mobility indices dropped again in October, as a consequence of governmental social-distancing policies instituted for the second pandemic wave (Fig. 1d). Using UV-index and mobility indices, we evaluated how seasonal factors and social distancing measures influenced the COVID-19 transmissibility, fitting a GAM model.

\section{Lag-effect analysis}

The effect of environmental and mobility indices variation on $R_{t}$ was not straightforward, as shown in Table 1. A GAM model including UV-index and atmospheric pressure displayed the best predictive effect on $\mathrm{R}_{\mathrm{t}}$ considering a lag-effect of two weeks, whereas temperature and humidity had the best predictive performance with a biologically unconvincing zero-week lag. Temperature and UV-index both displayed a definite seasonal variation and a strong correlation between each other, considering a four-week lag for temperature (Pearson coefficient 0.97) (Fig. 1b). 
Table 1

Lag effect analysis of influences on $R_{t}$. Each variable was evaluated at different lag times to explore potential plausible biological association with $\mathrm{R}_{\mathrm{t}}$. Numbers in boxes denote Akaike's information criterion (AIC) for that model. Lowest values mean a better fitting model. Models fitting best for positive week lags (1 week, 2 weeks) mean that explanatory variables preceded homologous $\mathrm{R}_{\mathrm{t}}$ fluctuations. * Represents the best fitting model for the selected variable.

\begin{tabular}{|lcll|}
\hline & O week & 1-week lag & 2-week lag \\
\hline UV-index & -8.15 & -9.23 & $-16.12^{*}$ \\
\hline Temperature & $-3.90^{*}$ & -0.54 & 0.37 \\
\hline Relative Humidity & $-3.25^{\star}$ & -0.69 & 0.03 \\
\hline Atmospheric Pressure & 0.85 & -0.85 & $-1.07^{*}$ \\
\hline Retail \& Recreation & -0.02 & -0.58 & $-0.69 *$ \\
\hline Grocery \& Pharmacy & -4.40 & -16.75 & $-18.87^{*}$ \\
\hline Workplaces & -3.63 & $-15.46^{*}$ & -12.01 \\
\hline Transit Stations & -1.02 & -3.11 & $-8.71 *$ \\
\hline Parks & $-8.09 *$ & -3.67 & 2.04 \\
\hline
\end{tabular}

The lag-effect of mobility indices variation differed. GAM models assessing Retail \& Recreation, Grocery \& Pharmacy and Transit Stations performed the best considering a lag-effect of two weeks, while Workplaces shows optimal performance with a one-week lag. Parks was the only mobility index to display the best performance considering an unconvincing zero-week lag.

Considering the AIC value (Table 1) and the predictable yearly dynamics of the UV-index, making it the fittest proxy for seasonality, the two-week UV-index lag was adopted for the main analysis, reflecting both the biological time needed for an external factor to influence transmissibility and for incubation time, as already shown by previous studies.[4, 5]

\section{Univariate analysis of influences on $R_{t}$}

The regression model with cubic splines was used to analyze the influences of each individual explanatory variable on $\mathrm{R}_{\mathrm{t}}$ and corresponding fitting degree of the model. According to our results, both seasonality and social distancing have a role in curbing the pandemic curve. The regression model used to analyze the influence of each individual mobility index on $R_{t}$ highlights a significant association with both UV-index and some mobility trends; all estimates and significance levels are listed in Table 2. In particular, the GAM including UV-index as single predictor variable explains $75.7 \%$ of $R_{t}$ deviance in Lombardy (adjusted $R^{2}=0.69 ; A I C=-13.40$ ). Concerning mobility indices, the highest explained deviance 
is $79.5 \%$ for the Grocery \& Pharmacy (adjusted $R^{2}=0.74 ; A I C=-18.87$ ). The lowest level of association was detected for Retail \& Recreation and Parks, with a deviance explained of $47.3 \%$ (adjusted $R^{2}=0.43$; AIC $=-0.69)$ and $44.7 \%$ (adjusted $\left.R^{2}=0.40 ; A I C=2.04\right)$, respectively; for these parameters, the $p$-value of the smoother resulted not significant.

Table 2

Univariate analysis of influences on $\mathbf{R}_{\mathbf{t}}$ : Each model is evaluated at the pre-determined 2-week lag. UV-index and Grocery \& Pharmacy based models display the best performances in predicting $\mathrm{R}_{\mathrm{t}}$ variations. Parks and Retail \& Recreation based models result as statistically non-significant. Edf; effective degrees of freedom; Ref.df: reference degrees of freedom; Dev exp: percent of deviance explained; AdjR2: adjusted R-squared; AIC: Akaike's information criterion.

\begin{tabular}{|lcccccccc|}
\hline & Edf & Ref.df & F-value & $\boldsymbol{p}$-value & Dev.exp & AdjR2 & AIC \\
\hline UV-index & 4.76 & 5.58 & 4.21 & 0.010 & 75.7 & 0.69 & -13.40 \\
\hline Retail \& Recreation & 1.00 & 1.00 & 1.28 & 0.270 & 47.3 & 0.43 & -0.69 \\
\hline Grocery \& Pharmacy & 4.46 & 5.25 & 6.39 & 0.001 & 79.5 & 0.74 & -18.87 \\
\hline Workplaces & 2.98 & 3.61 & 5.21 & 0.005 & 71.0 & 0.67 & -12.01 \\
\hline Transit Stations & 6.28 & 6.81 & 3.18 & 0.029 & 74.2 & 0.65 & -8.71 \\
\hline Parks & 1.00 & 1.00 & 0.04 & 0.850 & 44.7 & 0.40 & 2.04 \\
\hline
\end{tabular}

\section{Multivariate analysis of influences on $R_{t}$}

Multivariate GAM models of $\mathrm{R}_{\mathrm{t}}$ incorporating time-series correction, $\mathrm{UV}$-index, and mobility indices taken individually, considering a predetermined lag-effect of two weeks, were applied. All estimates and significance levels are listed in Table 3. 
Table 3

Multivariate analysis of influences on $\mathbf{R}_{\mathbf{t}}$ : Each model is evaluated at the pre-determined + 2week lag, and consists of mobility indices individually added to the model together with UVindex. Grocery \& Pharmacy, Transit Stations and Workplaces based models confirms themselves to be the most impactful predictors of $R_{t}$ variations, when weighted for seasonality. Edf; effective degrees of freedom; Ref df: reference degrees of freedom; Dev.exp: percent of deviance explained; AdjR2: adjusted R-squared; AIC: Akaike's information criterion.

\begin{tabular}{|lccccccc|}
\hline & Edf & Ref df & F-Value & $p$-Value & Dev.exp & AdjR2 & AIC \\
\hline UV-index & 4.76 & 5.58 & 4.21 & 0.007 & 75.7 & 0.69 & -13.40 \\
\hline Retail \& Recreation & 1.00 & 1.00 & 1.10 & 0.310 & 76.0 & 0.69 & -12.60 \\
\hline Grocery \& Pharmacy & 4.37 & 5.15 & 6.66 & 0.001 & 85.0 & 0.79 & -23.75 \\
\hline Workplaces & 2.64 & 3.19 & 4.08 & 0.019 & 82.6 & 0.76 & -19.88 \\
\hline Transit Stations & 6.46 & 6.80 & 4.00 & 0.010 & 85.5 & 0.78 & -20.19 \\
\hline Parks & 1.00 & 1.00 & 1.65 & 0.210 & 77.4 & 0.70 & -13.84 \\
\hline
\end{tabular}

In the aforementioned multivariate setting, Grocery \& Pharmacy displayed the best correlation with the response variable, with $85.0 \%$ deviance explained (adjusted $\mathrm{R}^{2}=0.79$; $\mathrm{AIC}=-23.75$ ). A reduction in the AIC score was also recorded for Workplaces (adjusted $\mathrm{R}^{2}=0.76 ; \mathrm{AIC}=-19.88$ ) and Transit Station (adjusted $R^{2}=0.78 ; A I C=-20.19$ ).

GAM multivariate results are shown in (Fig. 2a, 2b, 2c) as the smoother components plot for UV-index and each mobility index taken individually. Our model displays a steep downward curb in $\mathrm{R}_{\mathrm{t}}$ curve when Workplaces and Grocery \& Pharmacy shift in the $-20 \%$ to $-30 \%$ and -5 to $-15 \%$ interval, respectively, net of seasonality. Transit Station mobility index has a more complex association pattern, reflecting the degrees of freedom for the smoothing spline $(=6.46)$. However, a trend towards $R_{t}$ reduction in the $-20 \%$ to $-60 \%$ interval is still observable. All of the displayed models show a net effect of mobility indices in curbing $R_{t}$, despite seasonality. Our models contain only one mobility index per each, preventing us from evaluating the overall effect of UV-index on $\mathrm{R}_{\mathrm{t}}$, controlling for mobility.

\section{Discussion}

To the best of our knowledge, this is the first study to investigate the combined interplay of environmental and social interaction factors in SARS-CoV-2 transmissibility. In fact, one of the uncertainties regarding future reopening is the possibility for the pandemic to keep a near-stable seasonal profile, flattening in summer and exacerbating during the cold season, similarly to what has been observed in several midlatitude regions during 2020.[20] The results inferred by our data might be helpful in evaluating the timing and stringency of future interventions. 
Multiple factors may drive seasonal trends in respiratory viral infections, including change in social dynamics and intrinsic susceptibility of viruses to weather and environmental factors.[21] Indeed, using publicly available empirical data, collected for one of the most hardly hit regions in Europe[2] we explored multiple variables as possible contributors to a seasonal trend in SARS-CoV-2 transmissibility, namely mobility indices, solar UV radiation, temperature, atmospheric pressure, and humidity, assessing their effect with different time lags, in order to find the best fitting framework for our model. As already established by previous works, the biological effect carried out by external factors on case growth is not immediate.[4,5,22] Among selected environmental variables, UV-index displayed the strongest and the most plausible biological temporal correlation with $\mathrm{R}_{\mathrm{t}}$, making it the fittest to explain a putative environmental susceptibility of SARS-CoV-2.

According to our model, solar UV radiation, rather than temperature, might represent the key environmental factor contributing to SARS-CoV-2 transmissibility, accounting for $75.7 \%$ of $R_{t}$ deviance at the univariate analysis, holding a non-linear effect. The germicidal role of solar UV radiation (mainly UV-A and UV-B wavelengths) has already been explored, and recent experimental data suggests that solar UV exposure of a mid-latitude site during summer may lead to SARS-CoV-2 inactivation in the span of minutes. $[23,24]$ Solar UV exposure also plays a known and crucial role in the synthesis of vitamin $D$, which has a modulating effect on the immune response.[25] At the same time, epidemiological data exist supporting a negative association between COVID-19 transmissibility, severity, and UV radiation.[14,26,27]

On the other hand, social distancing policies have been implemented in many countries as the main countermeasure to the pandemic flood, and their association with SARS-CoV-2 transmissibility has been already established in several studies, using different proxies for the estimate.[4,5,28] In our study, we decided to consider mobility data per se, instead of focusing on the deployment of the policies that drove the drop in aggregate mobility values. The effect of specific restrictive interventions, evaluated by previous studies, was not assessed in the present study. Our model confirms that mobility plays a fundamental role in SARS-CoV-2 transmissibility, independently from environmental factors. Google Community Mobility Reports provide open-source data and have already proved to be useful in predicting COVID-19 case incidence.[29]

According to our analysis, Grocery \& Pharmacy, Transit Station and Workplaces predicted a considerable amount of $\mathrm{R}_{\mathrm{t}}$ variance, when taken individually, and maintain their significance when coupled with UVindex in a multivariate analysis. Conversely, Parks showed little-to-no effect on viral transmissibility. Surprisingly enough, Retail \& Recreation did not show a significant role in transmissibility either. This counterintuitive result, despite a similar graphical pattern between Retail \& Recreation and the aforementioned significant indices, may be due to an intrinsic feature of our dataset. In fact, similar relative changes in two indices may imply great differences in the absolute number of people actually involved, depending on the selected index. A small relative variation toward heavily frequented POls may have a more meaningful impact on $\mathrm{R}_{\mathrm{t}}$ when compared to a bigger relative variation of less frequented POls. This result is particularly meaningful in the light of the heavily implemented governmental bans on social events, restaurants closures, and restriction to leisure activities in general. 
Our model showed a strong correlation between social distancing policies (proxied by mobility patterns), seasonal environmental factors (proxied by UV-index), and fluctuation of SARS-CoV-2 transmissibility. This correlation was even stronger when mobility indices and UV-index were both included in the model, accounting for up to $85.5 \%$ of $R_{t}$ variance, thus suggesting their key role in transmissibility.

Our study has several limitations. First, due to the weekly calculation of $R_{t}$ estimate, the examined period of 30 weeks might imply low sampled data, affecting the model predictive value at the extremes of our curves, as shown by the large confidence intervals (Supplementary Figure 3). Moreover, due to the cyclical nature of some of the selected variables, an eight-month period may have limited our observable range for these factors. However, considering UV-index, due to an observation period roughly covering the time span between both solstices, a broad estimate of its whole seasonal half-cycle is expected to be included in our analysis. Second, we performed our analysis on a relatively small scale, so that further studies are needed to evaluate its generalization on a national or global scale. However, small-scale analysis has the advantage to limit data heterogeneity. In fact, nation-scale data averages may hide parameter variations occurring locally; conversely, an extreme variation in a spatially distinct smaller subsection can disproportionately sway a larger region's mean value. Third, the chosen mobility dataset has intrinsic limitations: it does not stratify for different demographics, missing a possible heterogeneity in viral susceptibility among different populations; it does not contain all POIs, in particular schools may be the great absentee from our dataset, being often debated as a source of infection spreaddoes not consider inter-regional travels. However, due to the capillary diffusion of Google Maps and Location Services among general population, Google Community Mobility Reports may represent the most meaningful and all-encompassing mobility data aggregator available to date; therefore, we expect our assumption based on this open-source service to hold robustly. Fourth, $\mathrm{R}_{\mathrm{t}}$ has intrinsic limitations that must be acknowledged.[30] However, since relatively few data are needed for its calculation and due to its general reliability, $R_{t}$ is universally considered a reliable index for the measurement of transmissibility in epidemics and was therefore adopted as our main response variable.

In conclusion, our results emphasize the importance of social distancing policies to keep viral transmissibility under control, especially during colder months. According to our analysis, smart-working policies, online grocery shopping, and avoidance of public transport overcrowding may be the most valuable measures to apply to contain case growth. UV-index displayed the best predictive value for SARS-CoV-2 transmissibility among the investigated environmental factors. With the coming of summer, which may mitigate future case growth, relaxations on mobility restriction, especially now that a new vaccine era has begun, may be considered. Further studies are needed to confirm our results on a more extended time and geographical scale.

\section{Declarations}

\section{ACKNOWLEDGMENTS}


We acknowledge Istituto Superiore di Sanità (ISS), the Emission Monitoring Internet Service (TEMIS) archive, NASA Prediction Of Worldwide Energy Resources (POWER) Data Access Viewer and Google Community Mobility Reports for their publicly available and detailed data which were used to inform our analysis.

\section{FUNDINGS}

The authors did not receive any specific funding for this manuscript.

\section{CONFLICTS OF INTEREST}

MF receives funding from Biogen Idec, Merck-Serono, Novartis, Roche, Teva Pharmaceutical Industries, outside the submitted work. MF receives compensation for consulting services and/or speaking activities from Almiral, Alexion, Bayer, Biogen, Celgene, Eli Lilly, Genzyme, Merck-Serono, Novartis, Roche, Sanofi, Takeda, and Teva Pharmaceutical Industries, outside the submitted work. MF is Editor-in-Chief of the Journal of Neurology and Associate Editor of Human Brain Mapping. YMF, LB, GS, TR and MV declare no competing interests.

\section{AVAILABILITY OF DATA AND MATERIALS}

All data used in the current study are available on the above mentioned open-source websites

\section{AUTHORS CONTRIBUTIONS}

YMF and LB conceived the study. LB and TR contributed to acquisition of data. GS and MV designed the model and conducted the analyses. YMF, LB and TR contributed to initial drafting of the manuscript. MF contributed to study supervision. All authors had access to all the data in the study, contributed to manuscript writing, and approved the final version.

\section{References}

1. WHO. Rolling updates on coronavirus disease (COVID-19). Available from: https://www. who.int/emergencies/diseases/novel-coronavirus-2019/events-as-they- happen (accessed December 20, 2020).

2. Remuzzi A, Remuzzi G. COVID-19 and Italy: what next? The Lancet. 2020;395:1225-8.

3. Google - COVID-19 Community Mobility Reports. Available from: https://www.google.com/covid19/mobility/ (accessed December 20, 2020).

4. Badr HS, Du H, Marshall M, Dong E, Squire MM, Gardner LM. Association between mobility patterns and COVID-19 transmission in the USA: a mathematical modelling study. The Lancet Infectious Diseases. 2020;20:1247-54.

5. Li Y, Campbell H, Kulkarni D, Harpur A, Nundy M, Wang X, et al. The temporal association of introducing and lifting non-pharmaceutical interventions with the time-varying reproduction number 
(R) of SARS-CoV-2: a modelling study across 131 countries. The Lancet Infectious Diseases. 2020;S1473309920307854.

6. Rader B, Scarpino SV, Nande A, Hill AL, Adlam B, Reiner RC, et al. Crowding and the shape of COVID19 epidemics. Nat Med. 2020;26:1829-34.

7. Cacciapaglia G, Cot C, Sannino F. Second wave COVID-19 pandemics in Europe: a temporal playbook. Sci Rep. 2020;10:15514.

8. Yap TF, Liu Z, Shveda RA, Preston DJ. A predictive model of the temperature-dependent inactivation of coronaviruses. Appl Phys Lett. 2020;117:060601.

9. Schuit M, Ratnesar-Shumate S, Yolitz J, Williams G, Weaver W, Green B, et al. Airborne SARS-CoV-2 Is Rapidly Inactivated by Simulated Sunlight. The Journal of Infectious Diseases. 2020;222:564-71.

10. Goh GK-M, Dunker AK, Foster JA, Uversky VN. Shell disorder analysis predicts greater resilience of the SARS-CoV-2 (COVID-19) outside the body and in body fluids. Microb Pathog. 2020;144:104177.

11. Bashir MF, Ma B, Bilal, Komal B, Bashir MA, Tan D, et al. Correlation between climate indicators and COVID-19 pandemic in New York, USA. Science of The Total Environment. 2020;728:138835.

12. Ma Y, Zhao Y, Liu J, He X, Wang B, Fu S, et al. Effects of temperature variation and humidity on the death of COVID-19 in Wuhan, China. Science of The Total Environment. 2020;724:138226.

13. Tosepu R, Gunawan J, Effendy DS, Ahmad LOAI, Lestari H, Bahar H, et al. Correlation between weather and Covid-19 pandemic in Jakarta, Indonesia. Science of The Total Environment. 2020;725:138436.

14. Isaia G, Diémoz H, Maluta F, Fountoulakis I, Ceccon D, di Sarra A, et al. Does solar ultraviolet radiation play a role in COVID-19 infection and deaths? An environmental ecological study in Italy. Sci Total Environ. 2020;143757.

15. Carlson CJ, Gomez ACR, Bansal S, Ryan SJ. Misconceptions about weather and seasonality must not misguide COVID-19 response. Nat Commun. 2020;11:4312.

16. Cori A, Ferguson NM, Fraser C, Cauchemez S. A New Framework and Software to Estimate TimeVarying Reproduction Numbers During Epidemics. American Journal of Epidemiology. 2013;178:1505-12.

17. Guzzetta G, Merler S. Stime della trasmissibilità di SARS-CoV-2 in Italia [Internet]. 2020. Available from: https://www.epicentro.iss.it/coronavirus/open-data/rt.pdf

18. Zempila M-M, van Geffen JHGM, Taylor M, Fountoulakis I, Koukouli M-E, van Weele M, et al. TEMIS UV product validation using NILU-UV ground-based measurements in Thessaloniki, Greece. Atmos Chem Phys. 2017;17:7157-74.

19. The Royal Society. Reproduction number (R) and growth rate $(r)$ of the COVID-19 epidemic in the UK: methods of estimation, data sources, causes of heterogeneity, and use as a guide in policy formulation [Internet]. Available from: https://royalsociety.org/-/media/policy/projects/set-c/setcovid-19-R-estimates.pdf?la=en-GB\&hash=FDFFC11968E5D247D8FF641930680BD6 (accessed December 20, 2020). 
20. The Johns Hopkins Coronavirus Resource Center (CRC) [Internet]. Available from: https://coronavirus.jhu.edu/map.html (accessed December 20, 2020).

21. Mallapaty S. Why COVID outbreaks look set to worsen this winter. Nature. 2020;586:653-653.

22. Carleton T, Cornetet J, Huybers P, Meng KC, Proctor J. Global evidence for ultraviolet radiation decreasing COVID-19 growth rates. Proc Natl Acad Sci USA. 2021;118:e2012370118.

23. Herman J, Biegel B, Huang L. Inactivation times from 290 to $315 \mathrm{~nm}$ UVB in sunlight for SARS coronaviruses CoV and CoV-2 using OMI satellite data for the sunlit Earth. Air Qual Atmos Health [Internet]. 2020 [cited 2021 Jan 21]; Available from: http://link.springer.com/10.1007/s11869-02000927-2

24. Ratnesar-Shumate S, Williams G, Green B, Krause M, Holland B, Wood S, et al. Simulated Sunlight Rapidly Inactivates SARS-CoV-2 on Surfaces. The Journal of Infectious Diseases. 2020;222:214-22.

25. Prietl B, Treiber G, Pieber T, Amrein K. Vitamin D and Immune Function. Nutrients. 2013;5:2502-21.

26. Sehra ST, Salciccioli JD, Wiebe DJ, Fundin S, Baker JF. Maximum Daily Temperature, Precipitation, Ultraviolet Light, and Rates of Transmission of Severe Acute Respiratory Syndrome Coronavirus 2 in the United States. Clinical Infectious Diseases. 2020;ciaa681.

27. Cacho PM, Hernández JL, López-Hoyos M, Martínez-Taboada VM. Can climatic factors explain the differences in COVID-19 incidence and severity across the Spanish regions?: An ecological study. Environ Health. 2020;19:106.

28. Kraemer MUG, Yang C-H, Gutierrez B, Wu C-H, Klein B, Pigott DM, et al. The effect of human mobility and control measures on the COVID-19 epidemic in China. Science. 2020;368:493-7.

29. Sulyok M, Walker M. Community movement and COVID-19: a global study using Google's Community Mobility Reports. Epidemiol Infect. 2020;148:e284.

30. Adam D. A guide to R - the pandemic's misunderstood metric. Nature. 2020;583:346-8.

\section{Figures}



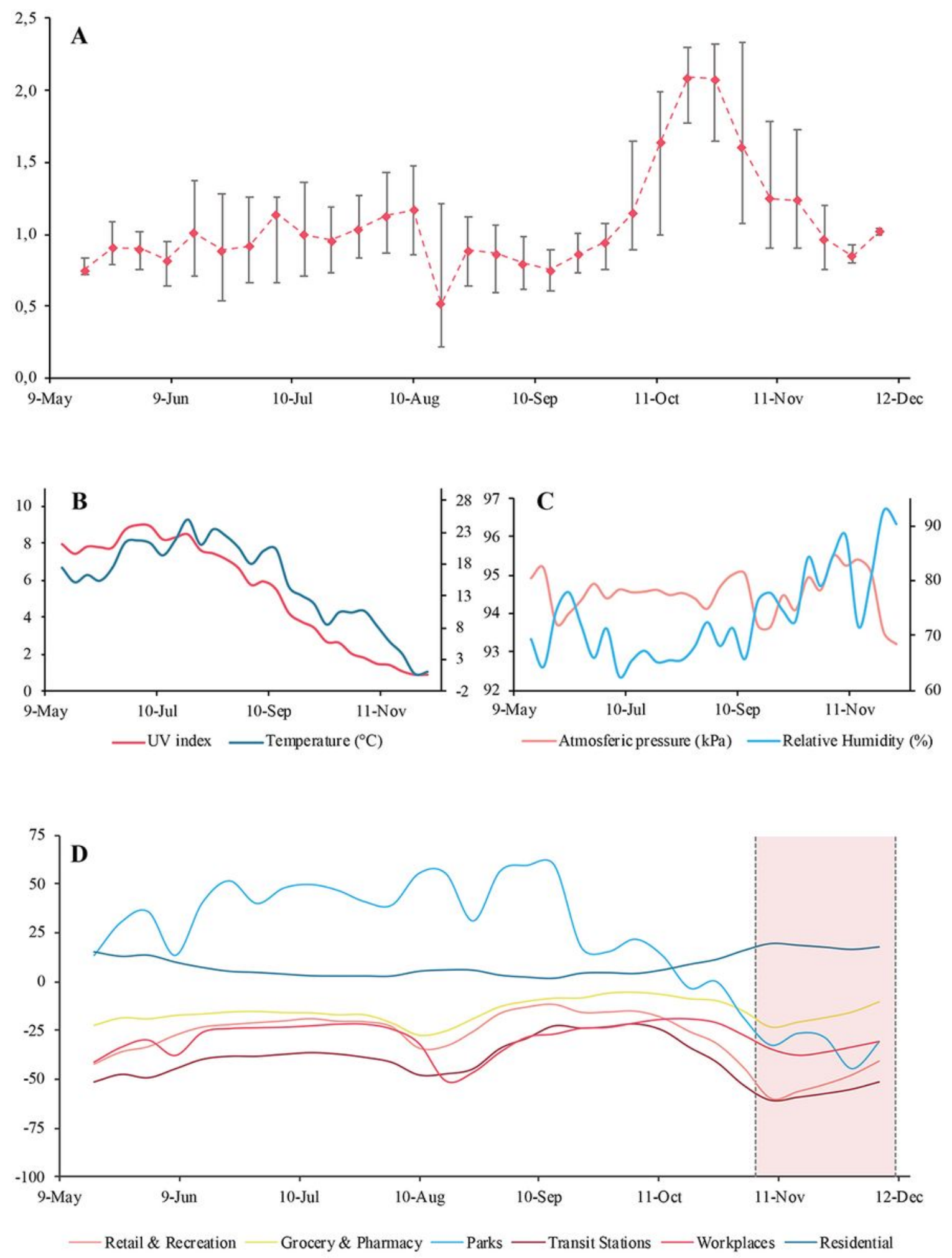

\section{Figure 1}

Observed trends in Rt and explanatory variables (A) Sampling of Rt values (95\% C.I.) and trend during the 30 weeks of our observation period. (B) UV-index (left axis) and temperature $\left({ }^{\circ} \mathrm{C}\right.$, right axis) trends. (C) Atmospheric pressure ( $\mathrm{kPa}$, left axis) and humidity (\%, right axis) trends. (D) Mobility indices trends in percent variation from baseline, considered individually. The red area between dashed vertical lines 
represents the introduction of "red zones" by the national government, defined by stay-at-home orders and strict restrictions to mobility implemented on regional basis.

A

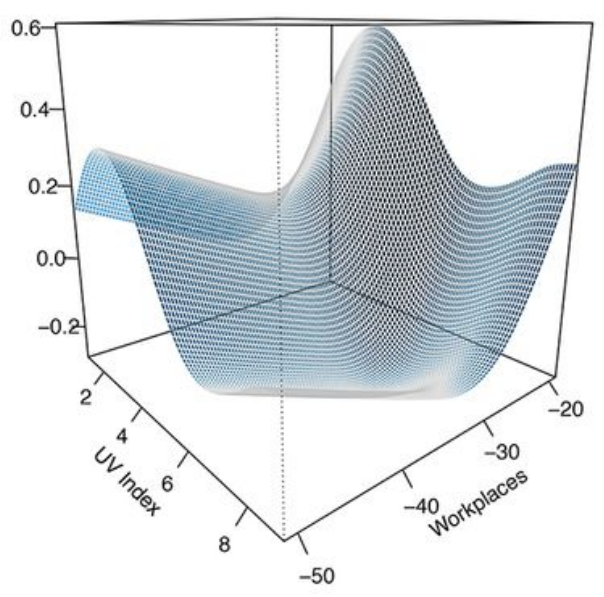

B

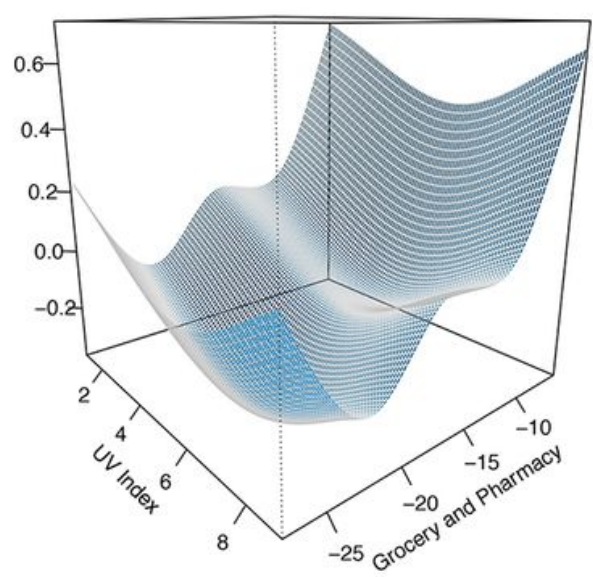

C

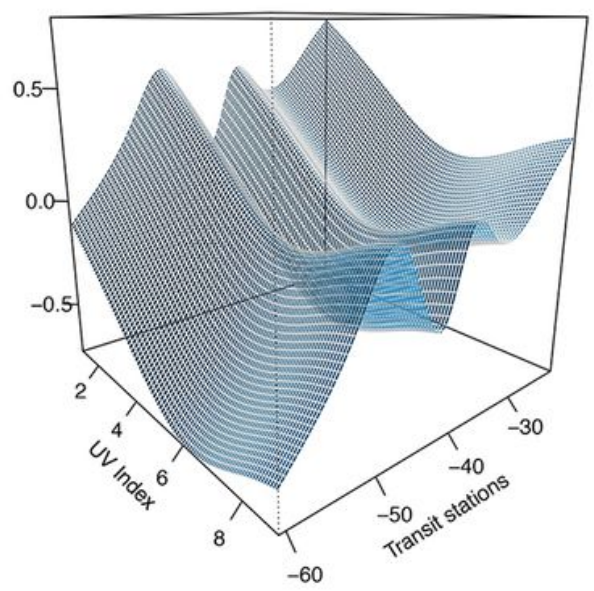

\section{Figure 2}

Three-dimensional effect graph of interacting influencing factors on the variation of $\log (\mathrm{Rt}) \mathrm{A}$ negative association between Workplaces (Fig.2A), Grocery \& Pharmacy (Fig.2B), and Transit Stations (Fig.2C) reduction and Rt (vertical axis)

\section{Supplementary Files}

This is a list of supplementary files associated with this preprint. Click to download.

- supplementarymaterial.docx 